\title{
Suivi De La Cinétique Du Développement Racinaire Des Plantes Destinées A La Stabilisation Des Talus Marneux De L'axe Autoroutier Fès-Taza (Nord Du Maroc)
}

\author{
Aicha Mouisat, \\ Institut Agronomique et Vétérinaire Hassan II (IAV), Rabat, Maroc \\ Ahmed Douaik,
Chaouki Al Faiz, \\ Institut National de la Recherche Agronomique (INRA), Rabat, Maroc \\ Chérif Harrouni, \\ Institut Agronomique et Vétérinaire Hassan II (IAV), Rabat, Maroc \\ Abdelkrim Derradji, \\ Société Nationale des Autoroutes du Maroc, Rabat, Maroc \\ Nour-Eddine Benaoda Tlemçani, \\ Institut Agronomique et Vétérinaire Hassan II (IAV), Rabat, Maroc
}

Doi:10.19044/esj.2020.v16n24p287 URL:http://dx.doi.org/10.19044/esj.2020.v16n24p287

Résumé

Le passage de l'autoroute Fès-Taza (Maroc) à travers des substrats marneux a engendré des talus particulièrement sensibles à l'érosion hydrique. En effet, sitôt confectionnés, ces talus subissent différentes formes d'érosion avec des sévérités variant avec l'intensité des pluies. Vu que la stabilisation mécanique s'est révélée difficile et trop coûteuse, les auteurs ont envisagé de faire appel au génie biologique mettant en œuvre les plantes qui contribuent au maintien du sol grâce à leur système racinaire. Ce travail concerne l'évaluation de l'effet, sur la stabilité du sol, de plusieurs espèces végétales plantées sur des talus marneux de l'axe autoroutier Fès-Taza. Des espèces prometteuses ont fait l'objet d'un suivi sous conditions contrôlées pour mieux comprendre la cinétique du développement de leurs racines. Il s'agit de: Acacia cyanophylla, Arundo donax, Medicago arborea, Prosopis pubescens, Retama monosperma et Spartium junceum. Ce suivi a été réalisé sur un substrat prélevé à partir des talus marneux de l'autoroute Fès-Taza et mis dans des mini-rhizotrons pour suivre la croissance et l'architecture du système racinaire. Acacia cyanophylla et Arundo donax, sont les espèces qui ont montré les densités racinaires les plus élevées. Medicago arborea est l'espèce qui a enregistré la vitesse moyenne d'allongement racinaire maximale parmi 
les espèces testées $(9,3 \mathrm{~cm} / \mathrm{mois})$. Ces six espèces ont montré des caractéristiques complémentaires nécessaires au maintien des talus. On les recommande comme composition floristique dans des conditions similaires.

Mots-clés: Autoroute, Erosion Hydrique, Génie Biologique, Sol Marneux, Système Racinaire, Talus

\title{
Kinetics Monitoring of Root Development of Some Plant Species Used for Soil Stabilization in the Embankments of the Fez-Taza Highway (North of Morocco)
}

\author{
Aicha Mouisat, \\ Institut Agronomique et Vétérinaire Hassan II (IAV), Rabat, Maroc \\ Ahmed Douaik, \\ Chaouki Al Faiz, \\ Institut National de la Recherche Agronomique (INRA), Rabat, Maroc \\ Chérif Harrouni, \\ Institut Agronomique et Vétérinaire Hassan II (IAV), Rabat, Maroc \\ Abdelkrim Derradji, \\ Société Nationale des Autoroutes du Maroc, Rabat, Maroc \\ Nour-Eddine Benaoda Tlemçani, \\ Institut Agronomique et Vétérinaire Hassan II (IAV), Rabat, Maroc
}

\begin{abstract}
Fez-Taza highway (Morocco) crosses a region characterized by dominant marly montainous soils which lead to many embankments, less or more susceptible to water erosion. These embankments become indeed subjected in subsequent years to various forms of erosion with varying severity depending on rain intensity. Since mechanical stabilization proved to be difficult and too costly, we decided to test some of the soil bioengineering technics, using some plant species, where root system could enables soil consolidation and thus reduces erosion. The purpose of this study is to evaluate the effect of several plant species, on soil stability. Plant species that showed promising results were closely monitored under controlled conditions to better understand the kinetics of their root development. These plants are: Acacia cyanophylla, Arundo donax, Medicago arborea, Prosopis pubescens, Retama monosperma and Spartium junceum. This monitoring was achieved under soil
\end{abstract}


substrate taken from the marly embankments of the Fez-Taza highway and filled into the mini-rhizotrons to follow growth and architecture of the root system. Acacia cyanophylla and Arundo donax are the species that showed the highest root densities. Medicago arborea is the specie that recorded the maximum mean of root elongation rate among the species tested $(9.3$ $\mathrm{cm} /$ month). These plant species have shown complementary characteristics necessary to maintain slopes. They are recommended as floristic composition under similar conditions.

Keywords: Highway, Water Erosion, Bio-Engineering, Marly Soil, Root System, Embankments

\section{Introduction}

L'utilisation de la végétation pour lutter contre l'érosion des sols représente une solution écologique, efficace et durable. Son importance et son efficacité ont été démontrées par plusieurs auteurs (Graf et al., 2003 ; Rey et al., 2004; Cammeraat et al., 2005 ; Norris et al., 2008 ; Lavaine et al., 2011; Mouisat et al., 2020). Avec une gestion adéquate, les communautés végétales peuvent protéger contre les dégradations du sol par érosion et par glissements de terrain peu profonds (Stokes et al., 2014). Cette protection est accomplie grâce à la canopée (partie aérienne) et au système racinaire.

Avec des parties aériennes bien développées, la végétation protège le sol contre l'effet cinétique des pluies par réduction de l'énergie des agents érosifs, empêchant ainsi l'ablation du substrat (Crosaz, 1994 ; Reiffsteck, 2003 ; Rey et al., 2004). Par l'interception des gouttes de pluie, le feuillage permet, d'une part, la soustraction d'une partie de l'eau qui tombe et d'autre part, la dissipation d'une partie de son énergie cinétique (Giordano, 1994 ; Rey et al., 2004). Mais l'efficacité de cette interception dépend de l'intensité de la pluie et de la densité du couvert végétal. Giordano (1994) a démontré qu'en forêt, les précipitations inférieures à $2 \mathrm{~mm}$ sont presque entièrement interceptées, tandis qu'au-delà de cette quantité, l'interception diminue selon une fonction logarithmique et pour une même essence l'interception augmente avec la densité et la surface foliaire. La canopée exerce aussi une action sur la perméabilité du sol et sur l'écoulement des eaux de ruissellement (Crosaz, 1994). La végétation agit donc comme une barrière protectrice entre le sol et les éléments naturels qui stimulent l'érosion (Stokes et al., 2008). Elle permet de réduire l'effet de battance jouant ainsi un rôle mécanique.

Les plantes diminuent l'énergie d'impact des eaux de précipitations atténuant ainsi l'érosion du sol supérieur (Graf et al., 2003). En plus du rôle d'interception, les plantes participent par leur feuillage dans le processus d'évapotranspiration par l'extraction de l'eau du sol (Graf et al., 2003 ; Stokes et al., 2014), ce qui permet d'en réguler le régime hydrique. Les tiges sont une 
composante très importante dans le soutien de la partie aérienne des plantes et elles interviennent aussi dans la lutte contre l'érosion en créant des obstacles retenant le sol érodé (Stokes et $a l$. , 2008) et réduisant la vitesse de ruissellement (Reiffsteck, 2003 ; Norris et al., 2008). Leur densité est un paramètre à prendre en considération pour l'évaluation du potentiel du piégeage (Stokes et al., 2014). En plus de la densité, la souplesse des tiges est intéressante pour la protection des sols. Lavaine et al. (2011) ont mis en évidence le rôle que jouent les tiges de Tamaricaceae grâce à leur souplesse qui leur permet de se coucher en cas de crue assurant ainsi une protection du sol par effet «tapis ». Elles permettent aussi de ralentir le courant, ce qui favorise le dépôt des sédiments.

Quant à la partie souterraine, la végétation permet de maintenir le sol grâce au système racinaire (Abernethy et Rutherfurd, 2000 ; Ammann et al., 2009 ; Erktan et al., 2018). En améliorant la cohésion des sols et en renforçant leurs propriétés mécaniques, les racines permettent d'en consolider et d'en renforcer la structure parce que la matrice sol-racine produit un complexe renforcé qui est beaucoup plus fort que le sol ou les racines séparément (Wu et al., 1979 ; Liu et Chen, 2015). La végétation améliore la structure du sol par l'enracinement et la production de litière et favorise l'activité biologique du sol. Cela entraîne des méso- et des macropores qui augmentent la capacité d'infiltration (Stokes et al., 2008). L'enracinement peut donc réduire les risques d'érosion en améliorant l'infiltration de l'eau et le drainage du sol (Drénou et al., 2006).

Outre le renforcement de la structure du sol, le système racinaire des plantes assure deux autres fonctions importantes : l'acquisition et le stockage des ressources hydriques et nutritionnelles ainsi que l'ancrage de la plante dans le sol (Fitter, 1987; Ennos, 2000 ; Mickovski, 2002 ; Drénou et al ., 2006). C'est l'architecture du système racinaire qui détermine la qualité de cet ancrage et la capacité de la plante à acquérir et transporter les ressources du sol (Watson et al., 1999 ; Dupuy, 2003 ; Genet et al., 2005 ; Abdi et al., 2010). Drénou et al. (2006) expliquent que, grâce à la combinaison qu'elles créent avec les éléments du sol, les racines augmentent considérablement l'ancrage des plantes en constituant une multitude de nœuds qui les fixent au sol. Le développement du système racinaire des plantes est primordial pour leur établissement et joue un rôle important dans la stabilité des pentes des sols vulnérables à l'érosion tels que les marnes (Burylo et al., 2010).

Même si les interactions végétation-érosion ont fait l'objet de plusieurs études, celles-ci restent peu développées en particulier dans le cas de l'érosion des talus routiers sur les marnes. L'objectif de cet article est d'améliorer les connaissances dans ce domaine, en se focalisant sur le rôle des racines des espèces végétales qui ont montré des résultats prometteurs pour la stabilisation des sols marneux dans des conditions topographiques (talus) et 
climatiques de la région que traverse l'axe autoroutier Fès-Taza du Maroc (Mouisat et al., 2020). Ces espèces ont été utilisées par le passé au sein de structures de génie biologique implantées sur les talus de l'autoroute. En effet, le génie biologique accompagne des aménagements d'une manière proche de la nature, se basant sur des matériaux de construction vivants, c'est-à-dire des semences, des plantes, des parties de végétaux et des associations végétales (Zeh, 2007).

\section{Materiel et methodes}

\subsection{Contexte de l'étude}

L'étude concerne les talus marneux de l'axe autoroutier Fès-Taza (126 $\mathrm{km}$ ) (Figure 1a), qui fait partie d'une zone montagneuse (entre 400-600 m d'altitude), faisant partie du massif du Pré-Rif. Cette zone est caractérisée par de forts épisodes pluvieux se concentrant sur quelques jours de la saison humide, s'étalant entre novembre et avril (Tableau 1). Ce sont des évènements qui donnent d'importantes quantités de pluie en quelques jours, voire en quelques heures. Ces précipitations interviennent directement dans les mécanismes d'érosion hydrique (Tribak et al., 2012). De fortes pluies sont d'ailleurs survenues pendant la période de notre étude (2014-2016), avec une intensité égale à $58 \mathrm{~mm} / \mathrm{h}$.

Tableau 1 : Répartition des précipitations durant la période allant de 2013 à 2017 (mm) (Station Bab Chhoub)

\begin{tabular}{|c|c|c|c|c|c|c|c|c|c|c|c|c|}
\hline Année & Jan & Fév & Mar & Avr & Mai & Juin & Juil & Aou & Sept & Oct & Nov & Déc \\
\hline 2013 & 206,4 & 96,6 & 289,0 & 59,3 & 35,3 & 0 & 0 & 0,5 & 15,8 & 0 & 115,3 & 66,4 \\
\hline 2014 & 116,0 & 78,0 & 121,2 & 35,0 & 6,8 & 6,6 & 0 & 0 & 0 & 20,2 & 69,5 & 93,7 \\
\hline 2015 & 115,2 & 69,8 & 53,6 & 14,0 & 71,2 & 2,0 & 0 & 0 & 8,2 & 25,8 & 9,8 & 0 \\
\hline 2016 & 29,6 & 99,8 & 67,0 & 26,4 & 53,4 & 1,2 & 3,6 & 2,2 & 17,0 & 41,4 & 101,5 & 162,6 \\
\hline 2017 & 52,2 & 60,8 & 21,0 & 7,4 & 7,0 & 0,4 & 0 & 16,8 & 0,8 & 22,2 & 83,8 & 78,4 \\
\hline
\end{tabular}

Les formes d'érosion observées sur les talus autoroutiers sont en général les griffes (profondeur de quelques centimètres (Roose, 1994)) et les rigoles (profondeur dépassant $10 \mathrm{~cm}$ (Roose,1994)) (Figure 1b,c). Le sol ainsi érodé se trouve accumulé progressivement en bas du talus, bouchant et saturant les caniveaux d'évacuation d'eaux et exposant la chaussée à des inondations pouvant gravement perturber la circulation. Les talus dénudés de toute végétation sont extrêmement vulnérables à cette érosion.

\subsection{Matériel biologique}

Pour lutter contre les différentes formes d'érosion observées sur les talus, des traitements basés sur la végétalisation implantée par des techniques de génie biologique (paillage à base de paille et roseaux, notamment) ont été envisagés. En effet, ces techniques utilisent des matériaux proches de la nature et permettent d'assurer un double rôle, à savoir la fixation de la paille par un 
fin grillage métallique fixé par des crochets en fer ou la fixation du paillage avec canisse de roseau, et l'ensemencement des talus.

Dans ce système et en vue d'assurer une durabilité de l'action protectrice, il faut étudier le développement du système racinaire en fonction des espèces végétales utilisées, pour une bonne stabilisation du sol des talus. Le choix des espèces à étudier s'est porté sur six espèces dont la liste et les caractéristiques sont mentionnées sur le tableau 2. Ces six espèces se sont montrées bien adaptées sur les talus marneux de l'autoroute Fès-Taza. (Mouisat et al.,2020) (Figure 1d)

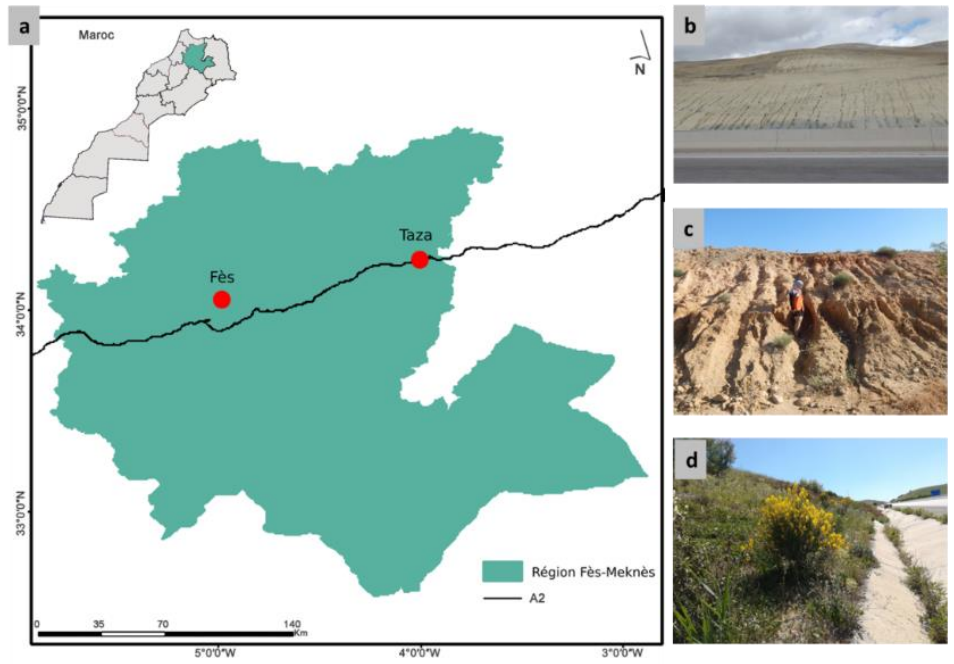

Figure 1: a: Situation de l'axe autoroutier Fès- Taza

b-c: Exemples des signes d'érosion observés sur l'axe autoroutier Fès-Taza $\mathrm{d}$ : Résultat après l'installation des techniques du génie biologique 


\begin{tabular}{|c|c|c|c|}
\hline Espèce & Famille & Origine & Quelques caractéristiques \\
\hline $\begin{array}{l}\text { Acacia } \\
\text { cyanophylla }\end{array}$ & Fabaceae & Australie & $\begin{array}{l}\text { Il s'agit d'un arbuste atteignant une dizaine de mètres de hauteur dans les conditions les plus } \\
\text { favorables (bords d'oued) et plus couramment de } 2 \text { à } 5 \text { mètres. Le port général est en boule. } \\
\text { C'est une essence qui possède des capacités d'adaptations intéressantes, elle peut être } \\
\text { plantée à peu près partout avec des chances de succès (Gillet, 1978). }\end{array}$ \\
\hline $\begin{array}{l}\text { Arundo } \\
\text { donax } \\
\text { (La canne de } \\
\text { Provence) }\end{array}$ & Poaceae & Méditerranéen & $\begin{array}{l}\text { Comme toutes les espèces du genre Arundo, la canne de provence se compose d'une partie } \\
\text { aérienne (chaumes) et d'une partie souterraine (rhizomes). Les rhizomes assurent la } \\
\text { croissance et classent les Arundo parmi les géophytes (Raunkiær 1934). A. donax s'établit } \\
\text { de préférence sur des sols nus et fortement humides, comme ceux des bords de parcelles } \\
\text { agricoles ou de ripisylves (Quinn et Holt, 2008).Il peut supporter un certain degré de } \\
\text { sécheresse expliquant son omniprésence dans le paysage méditerranéen (Hardion 2013). }\end{array}$ \\
\hline $\begin{array}{l}\text { Medicago } \\
\text { arborea } \\
\text { (la luzerne } \\
\text { arborescente) }\end{array}$ & Fabaceae & Méditerranéen & $\begin{array}{l}\text { C'est arbuste méditerranéen est considéré comme une espèce prometteuse dans les terres } \\
\text { arides et semi-arides où il peut jouer un rôle important dans l'élaboration d'un système } \\
\text { pastoral durable (Aissat et al., 2019). Il peut atteindre entre } 2 \text { et } 4 \mathrm{~m} \text { de hauteur dans les } \\
\text { conditions favorables (Guerrouj et al., 2013. Phylogénétiquement c'est la plus ancienne } \\
\text { espèce du genre Medicago et la seule avec un port arbustif (Martín et al., 2000). }\end{array}$ \\
\hline $\begin{array}{l}\text { Prosopis } \\
\text { pubescens } \\
\text { (Screwbean } \\
\text { Mesquite) }\end{array}$ & Fabaceae & Amérique du Nord & $\begin{array}{l}\text { C'est un petit arbre épineux qui peut atteindre une hauteur maximale de } 10 \mathrm{~m} \text { (Elias et } \\
\text { Dykeman, 1982). Facilement reconnaissable grâce à ses gousses en forme de vis (Little, } \\
\text { 1950). Comme toutes les espèces du genre Prosopis, cette espèce est importante pour la } \\
\text { végétalisation des milieux arides et semi arides. (Vilela et Ravetta, 2001) }\end{array}$ \\
\hline $\begin{array}{l}\text { Retama } \\
\text { monosperma } \\
\text { ( Retam) }\end{array}$ & Fabaceae & $\begin{array}{l}\text { Sud de l'Europe et pourtour du } \\
\text { bassin méditerranéen : Maroc, } \\
\text { Algérie, Égypte, Espagne } \\
\text { (Andalousie), Portugal, Italie, et } \\
\text { dans le désert sud asiatique. }\end{array}$ & $\begin{array}{c}\text { C'est une plante de } 2 \text { à } 4 \mathrm{~m} \text { de hauteur, connu pour ses fleurs ornementales. Elle est } \\
\text { psammophile (adaptée aux milieux sableux) (Le Houérou, 1995) et qualifiée de plante } \\
\text { fixatrice des dunes. }\end{array}$ \\
\hline $\begin{array}{l}\text { Spartium } \\
\text { junceum } \\
\text { (le genêt } \\
\text { d'Espagne) } \\
\end{array}$ & Fabaceae & Méditerranéen & $\begin{array}{l}\text { Arbrisseau de } 2 \text { à } 3 \mathrm{~m} \text { de hauteur (Tassin, 2012). Cette Papilionacée arbustive est caractérisée } \\
\text { par des inflorescences en grappes terminales indéfinies, sans fleur sans fleur terminale à } \\
\text { l'extrémité de certains rameaux. Presque tous les rameaux peuvent se terminer par une } \\
\text { inflorescence (Vescovi, 1964). C'est une espèce principalement calcicole, présente dans les } \\
\text { garrigues et les maquis, souvent liée à d'anciennes friches (Tassin, 2012). }\end{array}$ \\
\hline
\end{tabular}

Tableau 2 : Présentation des six espèces végétales suivies dans le mini rhizotron 


\subsection{Dispositif expérimental}

Le suivi de la croissance des racines a été assuré par des minirhizotrons pour une étude qualitative et quantitative du système racinaire. C'est une technique non destructive qui permet de suivre la dynamique de croissance des racines et leur architecture en visualisant leur répartition verticale et horizontale le long du profil du sol. Elle a été utilisée par plusieurs auteurs (Harrouni, 1989 ; Pagès, 1992 ; Glinski et al., 1993 ; Silva et Beeson, 2011).

Les mini-rhizotrons utilisés dans la présente étude sont sous forme de caissons en bois avec deux faces vitrées en retrait à la base par rapport à la verticale. Cette inclinaison permet aux racines en cours de croissance de se plaquer contre les vitres sous l'effet du géotropisme. Les parois vitrées sont recouvertes par un film en plastique noir pour assurer l'obscurité nécessaire aux racines et pour permettre l'observation et le suivi de leur développement. Les mini-rhizotrons ont été conçus selon les dimensions suivantes : 60x30x30 $\mathrm{cm}$ avec des trous de $0,5 \mathrm{~cm}$ de diamètre à la base pour le drainage. Une couche drainante composée de gravier est placée au fond des mini-rhizotrons. Dans le but de se rapprocher le plus possible des conditions naturelles, le substrat utilisé correspond à un échantillon prélevé d'un talus marneux, de texture argilo-limoneuse, de l'autoroute Fès-Taza en reconstituant les 6 premières couches du sol, chaque couche ayant une épaisseur de $10 \mathrm{~cm}$. Un arrosage a permis d'obtenir un échantillon compacté comparable à ce qui devrait se passer après un épisode pluvieux.

Il convient de souligner que 3 répétitions par espèce avec une seule plante par répétition, soit au total 3 plantes par mini-rhizotron ont eu lieu dans les mini-rhizotrons selon les dates suivantes :

- Le 22 Novembre 2014 (T0), pour les espèces végétales suivantes : la Arundo donax, Acacia cyanophylla, Spartium junceum et Retama monosperma.

- Le 20 Février 2015 (T0), pour les espèces suivantes : Prosopis pubescens et Medicago arborea.

Ces espèces ont montré une adaptation aux conditions édaphoclimatiques de la zone d'étude, et c'est sur cette base qu'elles ont été choisies pour suivre (in-vivo) l'évolution de leurs racines dans un dispositif rempli de substrat marneux. Pour mettre ces plantes dans des conditions similaires à celles installées sur les talus, on s'est contenté des eaux de pluie et on n'a pas apporté d'arrosage.

\subsection{Traits étudiés}

Le repérage et le comptage des racines ont été effectués à partir de différentes prises de vue des parois vitrées du mini-rhizotron. Les nouvelles 
apparitions des racines sont tracées pour chaque date de prise de vue à l'aide du logiciel de dessin Autocad. Les traits suivants ont été mesurés :

- Longueur racinaire $(\mathrm{cm})$ : c'est la longueur des apparitions racinaires ;

- Profondeur racinaire $(\mathrm{cm})$ : c'est la profondeur occupée par les apparitions racinaires;

- Vitesse d'allongement racinaire $(\mathrm{cm} / \mathrm{j}):(\mathrm{Pf}-\mathrm{Pi}) /(\mathrm{Tf}-\mathrm{Ti})$

Avec P: Profondeur racinaire et T: le Temps ; f : final ; i : initial.

En ce qui concerne le trait Hauteur aérienne $(\mathrm{cm})$ qui correspond à la hauteur de la partie aérienne, la mesure a été effectuée pendant les mêmes dates de prises de vues correspondant aux traits relatifs au système racinaire.

Le choix de l'étude des traits sur la longueur et la profondeur racinaires s'est fait dans le but de déterminer les espèces suceptibles d'offrir un maillage racinaire important, permettant ainsi la protection du sol et l'ancrage de la plante. En ce qui concerne la vitesse d'allongement racinaire, la détermination des espèces ayant une croissance racinaire rapide s'avère intéressante, en particulier dans la zone d'étude caractérisée par des conditions édaphoclimatiques difficiles. En effet, l'installation de telles espèces pourra aider à la fixation du sol des talus des tronçons nouvellement crées dans l'attente que les autres espèces avec croissance racinaire moins rapide puissent s'y proliférer. Le trait aérien a fait aussi l'objet de cette étude parce que c'est un critère qui renseigne sur la capacité de la plante à bien coloniser le sol et offrir une bonne couverture, et aussi parce que la canopée et le système racinaire sont liés.

Les dates de prises de vue sont les mêmes pour l'ensemble des espèces, elles ont été comprises entre le 10 février 2015 et le 14 avril 2016.

\subsection{Analyse statistique}

Les principaux paramètres statistiques descriptifs ont été calculés ainsi que la matrice des coefficients de corrélation entre la longueur des apparitions racinaires, la profondeur racinaire et la vitesse d'allongement racinaire des 6 espèces testées. La normalité des distributions des variables a été vérifiée en utilisant le test de Shapiro-Wilk alors que l'égalité des variances a été évaluée en utilisant le test de Levene. Pour les variables qui suivent une distribution normale, l'analyse de la variance (ANOVA) a été réalisée pour les deux variables «longueur des apparitions racinaires» et «hauteur de la partie aérienne» avec transformation logarithmique et correction de Welch pour la première variable et uniquement la correction de Welch pour la deuxième. L'ANOVA a été suivie du test de comparaisons multiples de moyennes de Duncan. Pour les variables qui ne suivent pas une distribution normale (profondeur racinaire et vitesse d'allongement racinaire), le test non paramétrique de Kruskal-Wallis a été utilisé pour détecter s'il existe une 
différence significative entre les espèces étudiées et le test de DunnBonferroni a été utilisé pour constituer des groupes homogènes d'espèces. Toutes les analyses statistiques ont été réalisées en utilisant le logiciel SAS 9.1.3, 2004.

\section{Resultats}

Les résultats présentés ci-dessous correspondent à l'évaluation du développement racinaire et aérien de six espèces végétales dans un sol marneux, dont les caractéristiques sont présentées dans le tableau 3.

Tableau 3 : Caractéristiques chimiques du sol utilisé dans l'expérience

\begin{tabular}{|c|c|c|c|c|c|}
\hline $\mathrm{PH}(\mathrm{eau})$ & $\mathrm{PH}(\mathrm{KCl} 1 \mathrm{~N})$ & $\mathrm{MO}(\%)$ & $\mathrm{P} 2 \mathrm{O} 5(\mathrm{ppm})$ & $\mathrm{K} 2 \mathrm{O}(\mathrm{ppm})$ & $\mathrm{CE}(\mathrm{mS} / \mathrm{Cm})$ \\
\hline 8,2 & 7,8 & 0,4 & 0,3 & 214,1 & 1,1 \\
\hline
\end{tabular}

\subsection{Longueur racinaire}

Durant la période de suivi de l'évolution des racines dans les minirhizotrons, c'est $A$. donax qui a la longueur racinaire moyenne la plus grande $(4 \mathrm{~cm})$, suivie par $P$. pubescens $(3,2 \mathrm{~cm})$ et A. cyanophylla $(2,9 \mathrm{~cm})$. Les trois autres espèces ont des longueurs racinaires moyennes inférieures à $2 \mathrm{~cm}$. La plus longue apparition racinaire a été enregistrée par $P$. pubescens avec 20,8 $\mathrm{cm}$. Le coefficient de variation pour l'ensemble des espèces est très élevé (CV $>50 \%$ ) allant de 56\% (S. junceum) jusqu'à 144\% (M. arborea), reflétant une très grande variabilité en ce qui concerne les longueurs des apparitions racinaires pour la même espèce et entre les espèces, ce qui nous donne une idée sur la dispersion et la morphologie racinaires.

Les résultats de l'ANOVA effectuée ont révélé des différences hautement significatives (Valeur de $F=31,1$ et $p<0,001$ ) pour l'effet des espèces étudiées sur la variable «Longueur racinaire», d'où le recours au test de comparaisons multiples de moyennes de Duncan (Figure 2). Ce test a révélé que: A. donax, $P$. Pubescens et $A$. cyanophylla ne sont pas significativement différentes entre elles $(\alpha=0,05)$, mais par contre, elles sont significativement différentes des autres espèces. Ces trois espèces ont les plus longues racines allant de 2,9 à $4 \mathrm{~cm}$. En revanche, les trois autres espèces non significativement différentes entre elles, ont les racines les plus courtes, comprises entre 1,1 et $1,7 \mathrm{~cm}$. 


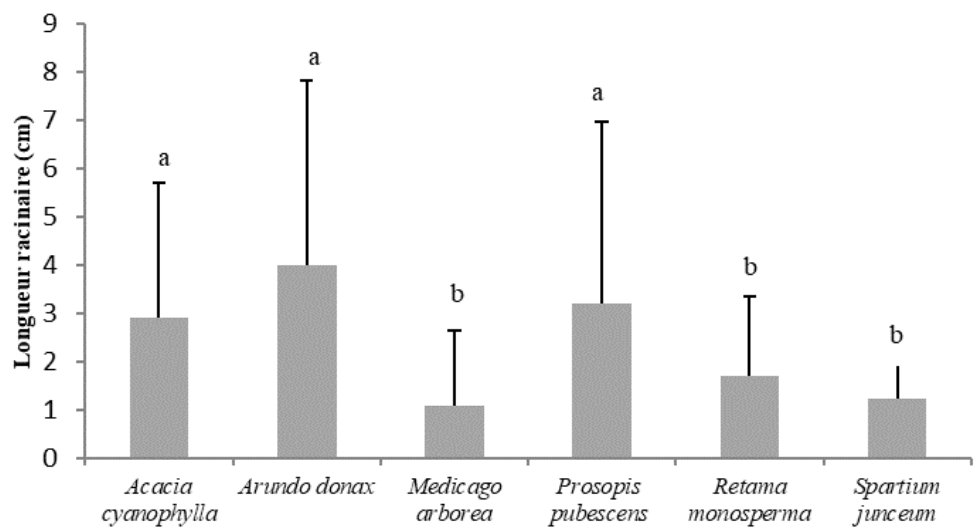

Figure 1 : Moyenne et écart type de la longueur racinaire des 6 espèces végétales testées dans le sol marneux. Les espèces qui possèdent les mêmes lettres ne sont pas significativement différentes entre elles $(\alpha=0,05)$, en utilisant le test de Duncan. Les histogrammes représentent les moyennes et les barres représentent les écarts types.

\subsection{Profondeur racinaire}

En ce qui concerne la profondeur racinaire, c'est $S$. junceum qui a enregistré la profondeur moyenne la plus intéressante, qui est de $38,3 \mathrm{~cm}$, suivie de R. monosperma, M. arborea, P. Pubescens, A. cyanophylla et $A$. donax avec des valeurs moyennes comprises entre 37,3 le cas du $R$. monosperma et $27,1 \mathrm{~cm}$ le cas de $A$. donax. A. cyanophylla a enregistré une profondeur maximale de $58,6 \mathrm{~cm}$ durant toute la période de suivi atteinte en 17 mois.

Le test non paramétrique de Kruskal-Wallis nous a permis de détecter qu'il existe des différences statistiquement significatives $(\alpha=0,05)$ en ce qui concerne la distribution de la variable «profondeur racinaire» pour les six espèces étudiées. Il reste de déterminer entre quelles espèces existent ces différences. C'est dans ce sens que le test de Dunn-Bonferroni pour les comparaisons multiples de médianes a été effectué (Figure 3). Les résultats de ce test ont mis en évidence qu'il existe une différence statistiquement significative entre $A$. donax et les autres espèces $(\alpha=0,05)$, qui n'ont pas montré de différences significatives entre elles. 


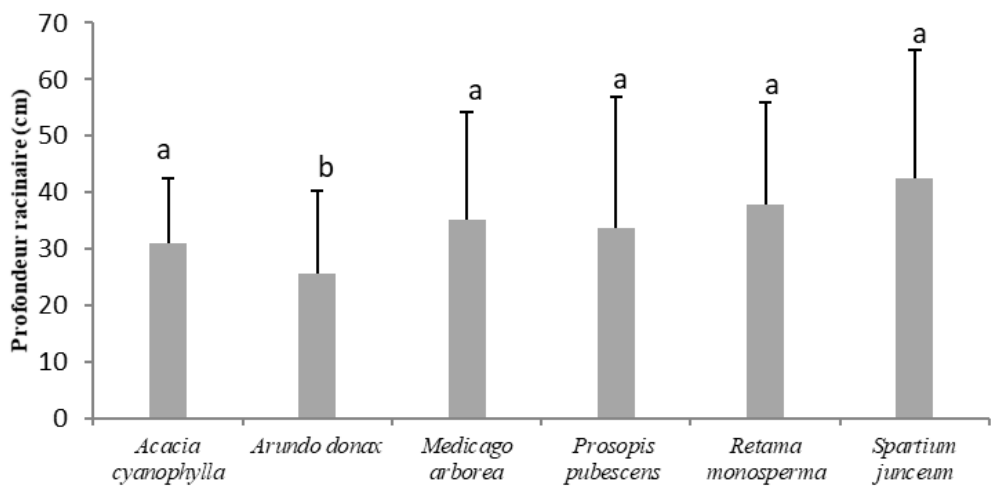

Figure 3 : Médiane et écart interquartile relatifs à la profondeur racinaire des 6 espèces végétales testées dans le substrat marneux. Test de Dunn-Bonferroni pour les comparaisons multiples de médianes. Les espèces qui possèdent les mêmes lettres ne sont pas significativement différentes ente elles $(\alpha=0,05)$. Les histogrammes représentent les médianes et les barres représentent l'écart interquartile.

\subsection{Vitesse d'allongement racinaire}

$M$. arborea est l'espèce qui a enregistré la vitesses moyenne la plus élevée en ce qui concerne l'allongement racinaire par rapport aux différentes espèces testées. Cette vitesse moyenne a été de $0,31 \mathrm{~cm} / \mathrm{j}$, soit presque 9,3 $\mathrm{cm} /$ mois. Pour les autres espèces les vitesses moyennes d'allongement racinaire ont été comprises entre 0,11 et $0,19 \mathrm{~cm} / \mathrm{j}$ en moyenne. Ceci correspond aux apparitions racinaires occupant des profondeurs inférieures ou égales à la profondeur initiale (Profondeur maximale des racines à T0 considérée comme profondeur initiale de référence). Les coefficients de variations correspondant à la variable «vitesse d'allongement racinaire» diffèrent d'une espèce à une autre.

Le test non paramétrique de Kruskal-Wallis nous a permis de détecter qu'il existe des différences statistiquement significatives $(\alpha=0,05)$ en ce qui concerne la distribution de la variable «vitesse d'allongement racinaire» pour les six espèces étudiées. Il reste de déterminer entre quelles espèces existent ces différences. C'est dans ce sens que le test de Dunn-Bonferroni pour les comparaisons multiples de médianes a été effectué (Figure 4). Ce test a révélé que $M$. arborea présente des différences statistiquement significatives avec toutes les autres espèces testées $(\alpha=0,05)$. En plus de cette espèce, $A$. donax et $P$. pubescens sont aussi statistiquement différentes concernant cette variable. 


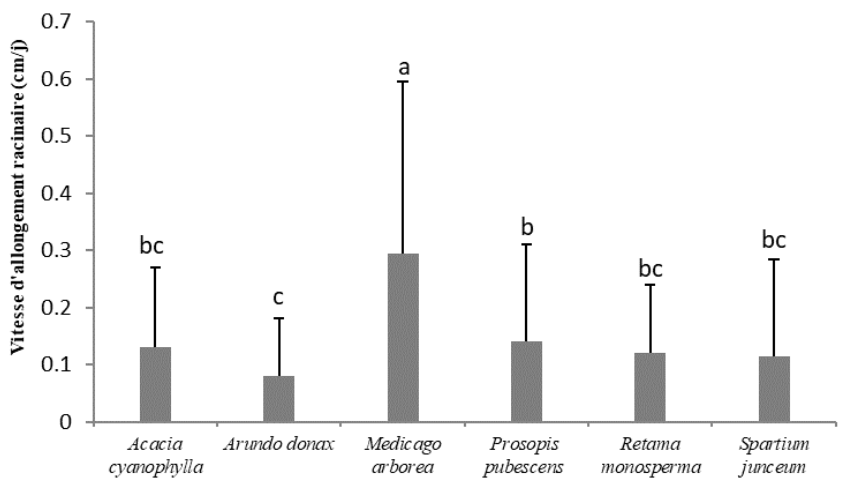

Figure 4: Médiane et écart interquartile relatifs à la vitesse d'allongement racinaire des 6 espèces végétales testées dans le substrat marneux. Test de Dunn-Bonferroni pour les comparaisons multiples de médianes. Les espèces qui possèdent les mêmes lettres ne sont pas significativement différentes entre elles $(\alpha=0,05)$. Les histogrammes représentent les médianes et barres représentent l'écart interquartile

\subsection{Corrélation des traits relatifs au système racinaire}

On prend l'exemple de $A$. donax et $P$. pubescens. Ces deux espèces ont présenté des systèmes racinaires assez différents dans le substrat marneux. La 1 ère espèce a été marquée par un chevelu racinaire très dense, tandis que pour la $2^{\text {ème }}$, les apparitions racinaires ont été minimes. Le test de Duncan n'a pas révélé de différence significative entre elles en ce qui concerne la longueur racinaire. Par contre, le test de Dunn-Bonferroni a montré qu'il existe des différences significatives entre elles pour les deux variables «profondeur racinaire» et «vitesse d'allongement racinaire». Pour comprendre les relations éventuelles entre ces variables, nous avons évalué la corrélation entre elles à l'aide du coefficient de corrélation de Spearman (Tableau 4).

Tableau 4 : Matrice de corrélation entre la longueur des apparitions racinaires, la profondeur racinaire et la vitesse d'allongement racinaire des six espèces testées (Coefficient de corrélation de Spearman, NS: non significatif ; *** $\mathrm{P}<0,001$ :très hautement significatif).

\begin{tabular}{|c|c|c|c|}
\hline & $\begin{array}{c}\text { Longueur } \\
\text { racinaire }\end{array}$ & $\begin{array}{c}\text { Profondeur } \\
\text { racinaire }\end{array}$ & $\begin{array}{c}\text { Vitesse d'allongement } \\
\text { racinaire }\end{array}$ \\
\hline Longueur racinaire & 1 & - & - \\
\hline Profondeur racinaire & $-0,07 \mathrm{NS}$ & 1 & - \\
\hline Vitesse d'allongement racinaire & $-0,23^{* * *}$ & $0,59^{* * *}$ & 1 \\
\hline
\end{tabular}

Les résultats des coefficients de corrélation montrent qu'il y a une corrélation très significative $(\mathrm{P}<0,001)$ entre, d'une part, la vitesse d'allongement racinaire et, d'autre part, la profondeur racinaire $(r=0,59)$ et la longueur racinaire $(-0,23)$. La corrélation négative indique que pour le cas des espèces testées et pendant la période de suivi, ce sont les petites ramifications racinaires occupant des profondeurs intéressantes qui ont eu un impact positif sur la vitesse d'allongement racinaire (Figure 5). C'est le cas de M. arborea qui est l'espèce qui a enregistré la vitesse maximale d'allongement racinaire 
et qui a un système racinaire marqué par plusieurs ramifications de petites longueurs dispersées sur les différentes couches du sol, y compris celles les plus profondes (Figure 5(c)). En revanche, A. donax est l'espèce qui a été marquée par la plus petite vitesse moyenne d'allongement racinaire, tandis qu'elle a des apparitions racinaires denses et de longueurs importantes, mais qui évoluent toutes en profondeur prenant ainsi plus de temps pour atteindre le fond des mini-rhizotrons.
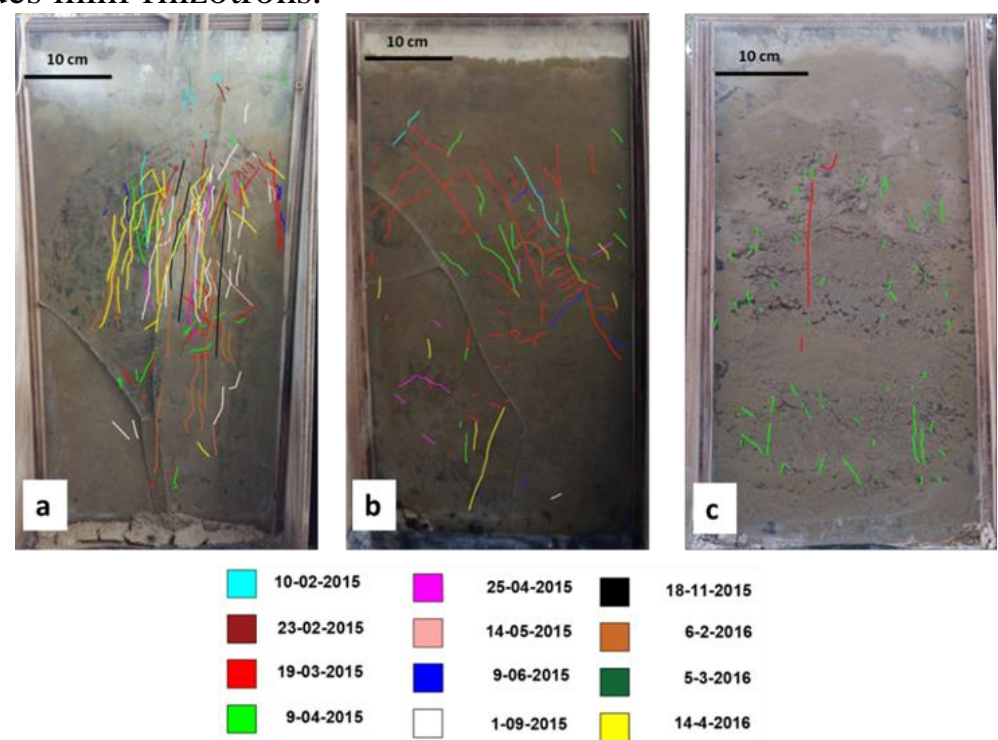

18-11-2015

6-2-2016

5-3-2016

14-4-2016

Figure 5: Distribution racinaire vue à partir des vitrées du mini-rhizotron (a) : A. donax (b) : A. cyanophylla - (c) : M. arborea. Les couleurs correspondent aux différentes dates de prises de vues.

\subsection{Hauteur de la partie aérienne}

En plus du suivi de l'évolution du système racinaire des espèces testées dans le substrat marneux, des relevés des hauteurs aériennes ont été effectués en fonction du temps pendant la période d'étude. A. cyanophylla est l'espèce qui a la hauteur aérienne maximale $(220 \mathrm{~cm})$, suivie d'A. donax avec une hauteur de $159 \mathrm{~cm}$. Les autres espèces ont enregistré des hauteurs aériennes comprises entre 63 et $87 \mathrm{~cm}$. Le même constat se fait en ce qui concerne les valeurs moyennes. La variation est modérée pour $M$. arborea, $P$. pubescens et $R$. monosperma avec des CV compris entre 20,5 et 29,6\%, alors qu'elle est forte pour les 3 autres espèces, avec des $\mathrm{CV}$ autour de $56 \%$.

L'ANOVA avec correction de Welch a été réalisée en vue de comprendre les différences éventuelles entre les espèces quant à leur hauteur aérienne. Les résultats de cette analyse montrent qu'il y a des différences significatives entre les six espèces étudiées (Valeur de $F=4,41$ et $p=0,002$ ), d'où le recours au test de comparaisons multiples de moyennes de Duncan (Figure 6). A. cyanophylla et A. donax n'ont pas présenté de différences 
significatives $(\alpha=0,05)$, ceci est dû à leurs hauteurs aériennes moyennes assez similaires $(91,4$ et 93,5 cm). Ces deux dernières espèces, caractérisées par les plus grandes hauteurs, diffèrent nettement de $S$. junceum, $M$. arborea et $P$. pubescens dont les hauteurs aériennes sont aussi assez similaires, variant entre 43,8 et $55,6 \mathrm{~cm}$. Finalement, $R$. monosperma a une hauteur intermédiaire entre les deux groupes d'espèces et ne présente pas de différence significative avec toutes les autres espèces testées.

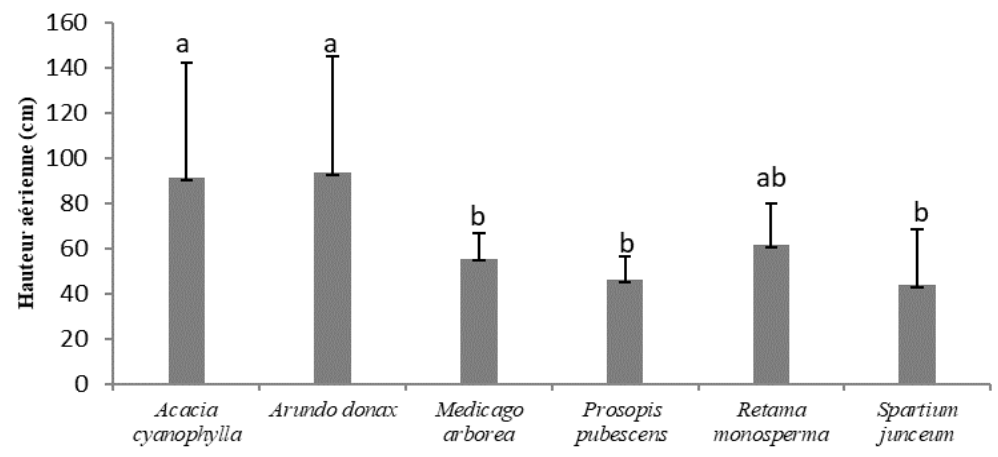

Figure 6 : Moyenne et écart type de la hauteur aérienne des 6 espèces végétales testées dans le sol marneux. Test de comparaisons multiples de moyennes de Duncan. Les espèces qui possèdent les mêmes lettres ne sont pas significativement différentes entre elles $(\alpha=0,05)$.

Les histogrammes représentent les moyennes et les barres représentent les écarts types.

\section{Discussion Et Perspectives}

Durant la période de suivi dans les mini-rhizotrons $A$. cyanophylla a enregistré des valeurs maximales pour la majorité des traits étudiés, que ce soit pour la partie souterraine ou bien pour la canopée. Ses racines ont franchi la profondeur maximale des mini-rhizotrons $(60 \mathrm{~cm})$ en presque 17 mois, ce qui devrait être plus en conditions naturelles puisque ses racines ont piqué dans le sol en dépassant la profondeur des mini-rhizotrons. Cette espèce a enregistré la profondeur maximale d'apparitions racinaires parmi les espèces étudiées. La profondeur racinaire est une caractéristique importante pour le maintien de l'arbre. Dans un peuplement planté de pin maritime (Pinus pinaster) âgés de 5 ans, les arbres les plus droits sont ceux qui ont la plus grande profondeur d'enracinement et la plus forte proportion de racines verticales et profondes (Danjon et al., 1999).

Les résultats émanant de cette étude ont montré que les deux espèces $S$. junceum et $R$. monosperma n'ont pas présenté de différences significatives $(\alpha=0,05)$ en ce qui concerne les différentes variables étudiées. S.junceum est l'espèce qui a enregistré la profondeur moyenne la plus élevée. Des études menées par Chiatante et al. $(2002 ; 2003)$ et Scippa et al. (2006) ont montré que les racines de $S$. junceum présentent des modifications pour s'adapter aux terrains en pente. Cette espèce renforce sa capacité d'ancrage en changeant l'organisation du système racinaire, en particulier dans la direction de la pente 
ascendante. L'architecture globale du système racinaire se modifie sur une pente avec une augmentation de la longueur racinaire et du nombre des ramifications. Preti et Giadrossich (2009) ont étudié les caractéristiques du système racinaire du genêt d'Espagne $(S$. junceum). Leurs résultats ont montré que cette espèce a de bonnes caractéristiques biomécaniques en ce qui concerne la stabilisation des pentes, même dans des conditions pédoclimatiques critiques et où les inclinaisons sont assez raides (Khuder, 2007). Ces résultats sont particulièrement intéressants dans le cas de cette présente étude, puisqu'il s'agit de talus marneux à renforcer dans l'ensemble du tronçon autoroutier. D'autant plus, c'est une espèce qui a fait preuve de bons résultats sur des terrains marneux (Marres, 1962 ; Karamitros, 1966 ; Chatain et Payany 1994 ; Delforge et al ., 2000 ; Mouisat et al., 2020). R. monosperma a enregistré une vitesse moyenne d'allongement racinaire de $0,11 \mathrm{~cm} / \mathrm{j}$ et une vitesse maximale de $0,28 \mathrm{~cm} / \mathrm{j}(\mathrm{CV}=61,7 \%)$. Cette dernière est inférieure à celles enregistrées par les autres espèces étudiées dans le substrat marneux. Néanmoins, les résultats observés sur le terrain en ce qui concerne son adaptation aux conditions climatiques de l'autoroute Fès-Taza sont très prometteurs. La plupart des études sur cette espèce ont porté surtout sur la bio-fertilisation, les nodules racinaires, la stabilisation des dunes, les symbioses végétales (Atbib, 1983 ; Muñoz-Vallés et al., 2010, 2013; Esquivias et al., 2014 ; Selami et al., 2014 ; Gallego-Fernández et al., 2015 ; Benelhadj-Djelloul, 2018), la phytostabilisation des résidus miniers (Lamin et al., 2019), la résistance au déficit hydrique (Fakhech et al., 2019) et à la salinité (Zunzunegui et al., 2017). L'étude de son rôle pour la lutte contre l'érosion des talus marneux reste peu développé. A partir des résultats de cette étude et des observations effectuées sur le terrain. C'est une plante à préconiser pour la stabilisation des talus autoroutiers marneux surtout en ce qui concerne les remblais.

Les racines de $M$. arborea ont montré une bonne adaptation au substrat marneux. C'est en effet l'espèce qui a enregistré la vitesse d'allongement racinaire la plus grande parmi les espèces étudiées. Cette espèce a présenté des différences significatives $(\alpha=0,05)$ en ce qui concerne la variable «vitesse d'allongement racinaire» par rapport aux cinq autres espèces. Elle est aussi significativement différente des trois espèces A. cyanophylla, A.donax et P.pubescens en ce qui concerne la variable « longueur racinaire ». Par ailleurs, pour la variable « profondeur racinaire », elle n'est significativement différente que de l'A. donax. En ce qui concerne la hauteur aérienne, cette espèce a fait preuve d'un comportement similaire aux espèces suivantes $: R$. monosperma, $P$. pubescens et $S$. junceum. Il a été montré que la luzerne arborescente ( $M$. arborea) est intéressante en ce qui concerne la restauration des terrains marneux (Andreu et al., 1994 ; Andreu et al., 1995 ; Roose, 2002 ; Morsli et Hamoudi, 2015 ; Mouisat et al., 2020). Elle a permis de réduire la 
production de sédiments de plus de $58 \%$ par rapport au sol nu (Andreu et al., 1998). Chebbi et al., (1994) ont trouvé que la longueur racinaire maximale pendant les 5 premières semaines de développement du $M$. arborea est de 69,4 $\mathrm{mm}$. On ne peut pas la comparer à celle trouvée durant cette étude puisque les conditions à $\mathrm{T}_{0}$ sont différentes. Néanmoins, à titre indicatif, on peut signaler que pendant les 7 premières semaines de croissance dans le substrat marneux, la longueur racinaire maximale de cette espèce est arrivée à $37,4 \mathrm{~cm}$ sachant qu'à $\mathrm{T}_{0}$ cette longueur était de $10 \mathrm{~cm}$.

A partir des résultats obtenus, $P$. pubescens a présenté des similarités avec certaines des espèces étudiées. Ces résultats, surtout ceux en relation avec la hauteur aérienne, sont inférieurs à ce qui a été attendu comme évolution aérienne en comparaison aux plants installés sur les talus de l'autoroute. Cette espèce joue un rôle important dans le contrôle de l'érosion (Pasiecznik et al., 2001 ; Zappala, 2012).

A. donax est caractérisée par le système racinaire le plus dense. Un constat normal puisque c'est une espèce caractérisée par des rhizomes puissants. Ses racines ont piqué dans le sol pendant la période de suivi dans les mini-rhizotrons, dépassant ainsi la profondeur de $60 \mathrm{~cm}$. C'est une plante qui a montré une bonne capacité pour prévenir l'érosion des terrains en pente (Christou et al., 2003). Dans des zones accidentées sujettes à l'érosion, elle a permis de réduire le ruissellement total de $61 \%$ par rapport à la jachère (Fagnano et al., 2014). Son réseau racinaire très dense protège bien le sol tandis que ses nombreuses tiges semi-rigides ralentissent les écoulements (Roose et al., 2010). Mais elle exige de très grandes quantités d'eau pour soutenir sa croissance rapide et vigoureuse (Nackley et al.,2014).

Les vitesses moyennes d'allongement racinaire des six espèces étudiées dans le substrat marneux sont légèrement inférieures à celles trouvées par Crosaz, (1995) en ce qui concerne quelques espèces colonisatrices des marnes noires.

La présente étude a mis en évidence différents types de comportement racinaire vis-vis du substrat marneux. Les six espèces étudiées se sont montrées adaptées aux conditions édapho-climatiques du site et ont développé des caractéristiques racinaires qui diffèrent d'une espèce à une autre et qui sont à aspect complémentaire permettant de contribuer à la stabilisation des talus marneux. Ceci est dû au comportement spécifique des racines de chaque espèce vis-à-vis du substrat marneux et de la capacité propre à chaque espèce de s'enraciner. En effet, Reubens et al. (2007) ont également suggéré qu'une combinaison de systèmes racinaires peu profonds mais denses et de racines grossières pénétrant profondément dans le sol pourrait être une solution efficace pour contrôler l'érosion.

Le dispositif utilisé dans cette expérimentation n'a permis le suivi des racines que dans une profondeur limitée de $60 \mathrm{~cm}$, ce qui laisse présager de 
l'évolution de la biomasse racinaire au-delà de cette profondeur. Une étude comparative avec des méthodes plus développées peut être envisagée. Ceci, en évaluant l'effet des pentes sur le développement de ces espèces et l'efficacité de ces espèces dans la lutte contre l'érosion selon différentes classes de pentes.

Les résultats de ce travail pourront servir à concevoir un dispositif expérimental plus développé, en approfondissant les recherches sur d'autres espèces végétales, et en comparant leurs réponses à différentes conditions édapho-climatiques, parce que les présents résultats ne peuvent être appliqués que dans la région Fès-Taza ou bien des zones à conditions similaires.

\section{Conclusion Et Recommandations}

Cette étude a mis l'accent sur le développement racinaire d'espèces qui se sont montrées adaptées sur les talus marneux. Les mesures et observations réalisées montrent que la croissance racinaire sur substrat marneux diffère d'une espèce à une autre. Ainsi, la vitesse moyenne d'allongement racinaire pour les espèces végétales conduites en mini-rhizotrons a atteint $9,3 \mathrm{~cm} / \mathrm{mois}$ pour $M$. arborea. C'est une vitesse qu'on peut juger intéressante vis-à-vis de ce type de sol contraignant. Les racines d'A. cyanophylla et d'A. donax ont développé un chevelu racinaire important. Ces deux espèces ont aussi montré une production de biomasse aérienne élevée. Ceci explique d'ailleurs les résultats sur racines, puisque la dynamique des deux systèmes est liée. Une plante vigoureuse a besoin d'un important système racinaire pour s'alimenter, et le système racinaire à son tour a besoin d'une canopée développée pour assurer sa croissance et sa fonction biologique.

Pour lutter contre l'érosion dans ce type de sol, il faudrait associer des espèces végétales avec des attributs complémentaires : des plantes à vitesse de croissance élevée pionnières pour protéger la couche superficielle du sol, le temps que des espèces ayant une densité racinaire plus importante, et une longévité plus élevée puissent s'installer et constituer l'armature du sol pour lutter de façon durable contre l'érosion. Dans cette perspective, nous recommandons les six espèces étudiées à raison des pourcentages suivants : Acacia cyanophylla : 20\%; Arundu donax : 10\% ; Medicago arborea : 15\% Prosopis pubescens : 20\% ; Retama monosperma : 15\% ; Spartium junceum : $20 \%$. En plus de ces espèces, on pourrait recommander l'ajout d'autres espèces telles que des Agaves et des Atriplex, deux espèces particulièrement résistantes à la sécheresse, pour améliorer la résilience du mélange floristique au niveau du talus.

\section{Remerciements}

Les auteurs souhaitent remercier $M^{\text {elle }}$ Rajae Hamoud, technicienne à l'INRA de Rabat, pour son aide en ce qui concerne la préparation des espèces 
végétales et M. Rachid Mohib pour l'espace dédié à l'installation des minirhizotrons.

\section{References:}

1. Abdi, E., Majnounian, B., Genet, M., \& Rahimi, H. (2010). Quantifying the effects of root reinforcement of Persian ironwood (Parrotia persica) on slope stability; a case study: Hillslope of Hyrcanian forests, northern Iran. Ecological Engineering, 36:14091416

2. Abernethy, B \& Rutherfurd, I.D. (2000). The effect of riparian tree roots on the mass-stability of riverbanks. Earth Surface Processes and Landforms, 25: 921-937

3. Aissat, A., Mehdadi, Z, Rita Leogrande, R., \& Stellacci, A M. (2019). Characterization of Medicago arborea L. Response to Water and Salt Stress. Journal of Stress Physiology \& Biochemistry, 15:103-113

4. Ammann, M., Böll, A., Rickli, C., Speck, T., \& Holdenrieder, O. (2009). Significance of tree root decomposition for shallow landslides. Forest Snow and Landscape Research. 82: 79-94

5. Andreu, V., Rubio, J.L., Gimeno- Garcia, E., \& Llinares, J.V. (1998). Testing three Mediterranean shrub species in runoff reduction and sediment transport. Soil and Tillage Research, 45: 441-454

6. Andreu, V., Rubio, J.L., \& Cerni, R. (1995). Effect of Mediterranean shrub on water erosion control. Environmental Monitoring and Assessment, 37:5-15

7. Andreu, V., Rubio, J.L., \& Cerni, R. (1994). Use of a shrub (Medicago arborea) to control water erosion on steep slopes. Soil Use and Management, $10: 95-99$

8. Atbib, M. (1983). Etude phytoécologique de la réserve biologique de Mehdia (littoral atlantique du Maroc), II La végétation du milieu dunaire. Bulletin de l'institut scientifique, Rabat, 7: $129 \mathrm{p}$

9. Benelhadj-Djelloul, S. (2018). Rôle des symbioses végétales dans la réhabilitation de la sablière de Terga (Wilaya d' Ain Temouchent. Thèse. Département de Biotechnologie. Université d'Oran1- Ahmed Ben Bella. 178p

10. Bischetti, G.B. (2009). Root cohesion of forest species in the Italian Alps. Plant and soil, 324:71-89

11. Burylo, M., Hudek, C., \& Rey, F. (2010). Soil reinforcement by the root system of six dominant species on eroded mountainous marly slopes (Southern Alps, France). CATENA, 84 :70-78

12. Cammeraat, E., Van Beek, R., \& Kooijman, A. (2005). Vegetation succession and its consequences for slope stability in SE Spain. Plant and soil, 278: 135-147 
13. Chatain, M. \& Payany, M. (1994). La végétalisation: outil d'aménagement, guide technique. Service d'études techniques des routes et autoroutes, $111 \mathrm{p}$

14. Chebbi, H., Ríos, S., Pascual, M.J., \& Correal, E. (1994). El grupo Medicago arborea en la Cuenca Mediterránea: II. Comportamiento frente a la sequía. Revista Pastos 24 : 177-188

15. Chiatante, D., Scippa, S.G., Di Iorio, A., \& Sarnataro, M. (2002). The influence of steep slopes on root system development. Journal of Plant Growth Regulation, 21:247-260

16. Chiatante, D., Sarnataro, M., Fusco, S., Di Iorio, A., \& Scippa, G. S. (2003). Modification of root morphological parameters and root architecture in seedlings of Fraxinus ornus L. and Spartium junceum L. growing on slopes. Plant Biosystems, 137: 47-55

17. Christou, M., Mardikis, M., Alexopoulou, E., Cosentino, S., Copani, V., \& Sanzone, E. (2003). Environmental studies on Arundo donax. Proceedings of the 8th International Conference on Environmental Science and Technology. Lemnos island, Greece. Pp: 102-110

18. Crosaz, Y. (1994). Le matériel végétal : un outil pour la protection des sols. Actes des onzièmes journées du Réseau Erosion, Centre ORSTOM de Bondy / Septembre 1994. 449-460

19. Danjon, F., Bert, D., Godin, C., \& Trichet, P. (1999). Structural root architecture of 5-year-old Pinus pinaster measured by 3D digitising and analysed with AMAPmod. Plant and Soil, 217: 49-63

20. Danquechin Dorval, A. (2015). Architecture racinaire et stabilité chez le pin maritime (Pinus pinaster Ait.) au stade jeune. Thèse. Université de Bordeaux. 205p

21. Delforge, P., Devillers-Terschuren, J., \& Devillers, P. (2000). L'Ophrys de Lucanie, Ophrys lucana, une espèce nouvelle du groupe d'Ophrys obaesa. Natural. Belges, 81 (Orchid. 13): 257-268

22. Drénou, C., Bonneau, M., Charnet, F., Cruiziat, P., Frochot, H., Garbaye, J., Girard, S., Larrieu, L., Lévy, G., Marçais, B., Moore, W., \& Rossignol, J.P. (2006). Les racines : face cachée des arbres. Institut pour le développement forestier CNPPF. 338p.

23. Dupuy, L. (2003). Modélisation de l'ancrage racinaire des arbres forestiers. Thèse. Université de Bordeaux I. 135p

24. Elias, T.S. \& Dykeman, Peter A. (1982). Field guide to North American edible wild plants. New York: Outdoor Life Books. $286 \mathrm{p}$

25. Ennos, A.R. (2000). The mechanics of root anchorage. Advances in Botanical Research 33: 133-157

26. Erktan, A., McCormack, ML., \& Roumet, C. (2018). Frontiers in root ecology: recent advances and future challenges. Plant Soil, 424:1-9 
27. Esquivias, M.P., Zunzunegui, M., Díaz Barradas, M.C., \& ÁlvarezCansino, L. (2014). Competitive effect of a native-invasive species on a threatened shrub in a Mediterranean dune system. Oecologia, 177 : 133-146

28. Fagnano, M., Impagliazzo, A., Mori, M., \& Fiorentino, N. (2014). Agronomic and Environmental Impacts of Giant Reed (Arundo donax L.) : Results from a Long-Term Field Experiment in Hilly Areas Subject to Soil Erosion. BioEnergy Research, 8: 415-422

29. Fakhech, A., Manaut, N., Ouahmane, L., \& Hafidi, M. (2019). Contributions of indigenous arbuscular mycorrhizal fungi to growth of retama monosperma and Acacia gummifera under water stress (case study: Essaouira sand dunes forest). Journal of Sustainable Forestry, 38: 686-696

30. Fitter, A. H. (1987). An architectural approach to the comparative ecology of plant root systems. New Phytologist, 106: 61-77

31. Gallego-Fernández, J.B., Muñoz-Valles, S., \& Dellafiore, C. M. (2015). Spatio-temporal patterns of colonization and expansion of Retama monosperma on developing coastal dunes. Journal of Coastal Conservation, 19: 577-587

32. Genet, M., Stokes, A., Salin, F., Mickovski, S., Fourcaud, T., Dumail, J.F., \& Van Beek, R. (2005). The influence of cellulose content on tensile strength in tree roots. Plant and Soil, 278: 1-9

33. Gillet, H. (1978). Un Acacia australien à la conquête des dunes de Libye. Journal d'agriculture traditionnelle et de botanique appliquée. (4): 245-255

34. Giordano, A. (1994). L'érosion et la lutte contre l'érosion en forêt méditerranéenne. Forêt méditerranéenne, 15 :12-21

35. Glinski, D. S., Karnok, K. J., \& Carrow, R. N. (1993). Comparison of reporting methods for root growth data from transparent-interface measurements. Crop Science, 33:310-314

36. Graf, C., Böll, A., \& Graf, F. (2003). Des plantes pour lutter contre l'érosion et les glissements en surface, Notice pour le praticien. $37: 8 \mathrm{p}$

37. Guerrouj, K., Pérez-Valera, E., Abdelmoumen, H., Bedmar, E. J., \& Missbah El Idrissi, M. (2013). Ensifer meliloti is the preferred symbiont of Medicago arborea in eastern Morocco soils. Canadian Journal of Microbiology, 59:540-548

38. Hardion, L. (2013). Evolution et systématique du genre Arundo L. (Poaceae), et conservation d'un endémique ligure : interactions Homme/Biodiversité en Méditerranée. Thèse. Aix-Marseille Université. $273 \mathrm{p}$ 
39. Harrouni, M.C. (1989). The effect of water stress on growth and development of barley and tef. Ph.D. thesis, University College of North Wales, Bangor, UK

40. Karamitros, E. (1966). Lutte contre l'érosion torrentielle en Grèce. Revue Forestière Française, 4: 266-280

41. Khuder, H. (2007). Effet d'une pente sur l'architecture et les propriétés mécaniques des systèmes racinaires de semis d'arbres. Thèse. Ecole Doctorale des Sciences Physiques et de l'Ingenieur. Université Bordeaux I. 182p.

42. Lamin, H., Alami, S., Bouhnik, O., El Faik, S., Abdelmoumen, H., Bedmar, E. J., \& Missbah-El Idrissi, M. (2019). Nodulation of Retama monosperma by Ensifer aridi in an abandonned lead mine soils in Eastern Morocco. Frontiers in Microbiology, 10: 1456.

43. Lavaine, C., Evette, A., Piegay, H., Lachat, B. \& Brahic, P. (2011). Les Tamaricaceae en génie végétal. Sciences, eaux et territoires, Revue d'IRESTEA, article hors-série, 4: 1-9

44. Liu, H \& Chen, Z. (2015). Debris flow disasters induced by forest firetaking the Reneyong gully as an example. International Forum on Energy, Environment Science and Materials (IFEESM 2015), 14041409

45. Le Houérou, H.N. (1995). Bioclimatologie et biogéographie des steppes arides du Nord de l'Afrique: diversité biologique, développement durable et désertisation. Options Méditerranéennes: Série B. Etudes et Recherches. Montpellier : CIHEAM, (1 0) :397p

46. Little, E.L. (1950). Southwestern trees: A guide to the native species of New Mexico and Arizona. Agric. Handb. 9. Washington, DC: U.S. Department of Agriculture, Forest Service. 109 p

47. Marres, P. (1962). Contribution à l'étude de la végétation de la région de Nîmes. Compte rendu. Méditerranée, $3^{\mathrm{e}}$ année, 1: 90-96

48. Martín, J. P., Pintos, B., Rebordinos, I., Villalobos, N., Guerra, H., \& Martín, L. (2000). Embryogenic Response in Different Medicago arborea L. Explants Depending on Cytokinin/Auxin Balances. Journal of Plant Physiology, 156: 801-804.

49. Mickovski, S. (2002). Anchorage mechanics of different types of root systems. Thèse, School of Biological Sciences, University of Manchester.149 p

50. Morsli, B., \& Hamoudi, A. (2015). Restauration des terres de parcours dans les Monts de Beni Chougrane (NO Algérie) : mise en défens et enrichissement en légumineuses ». In Roose, É. (éd.): Restauration de la productivité des sols tropicaux et méditerranéens, Montpellier, IRD Éditions, 9 p 
51. Mouisat, A., Harrouni, C., Al Faiz, C., Douaik, A., Derradji, A., \& Tlemçani Benaoda, N. (2020). Efficacité des méthodes du génie biologique pour lutter contre l'érosion hydrique des talus marneux de l'autoroute Fès-Taza. Revue Marocaine des Sciences Agronomiques et Vétérinaires. 8: 67-77

52. Muñoz Vallés, S., Gallego Fernández, J. B., \& Cambrollé, J. (2013). The biological flora of coastal dunes and wetlands: Retama monosperma (L.) Boiss. Journal of Coastal Research, 29:1101-1110

53. Muñoz-Vallés, S., Gallego Fernández, J. B., Dellafiore, C., \& Cambrollé, J. (2010). Effects on soil, microclimate and vegetation of the native-invasive Retama monosperma (L.) in coastal dunes. Plant Ecology, 212:169-179

54. Nackley, L. L.,Vogt, K. A., \& Kim, S.-H. (2014). Arundo donax water use and photosynthetic responses to drought and elevated $\mathrm{CO} 2$. Agricultural Water Management, 136: 13-22

55. Norris, J.E., Di Iorio, A, Stokes, A., Nicoll, B.C., \& Achim, A. (2008). Species Selection for Soil Reinforcement and Protection. Slope stability and erosion control, Ecotechnological solutions, 167-210

56. Pagès, L. (1992). Mini-rhizotrons transparents pour l'étude du système racinaire de jeunes plantes. Application à la caractérisation du développement racinaire de jeunes chênes (Quercus robus). Canadian Journal of Botany. 70:1840-1847

57. Pasiecznik, N.M., Felker, P., Harris, P.J.C., Harsh, L.N., Cruz, G., Tewar,i J.C., Cadonet, K., \& Maldonado, L.J. (2001). The Prosopis juliflora-Prosopis pallida Complex: A monograph. HDRA, Coventry, UK. $172 \mathrm{p}$

58. Preti, F., \& Giadrossich, F. (2009). Root reinforcement and slope bioengineering stabilization by Spanish broom (Spartium junceum L.). Hydrology and Earth System Sciences, 13: 1713-1726

59. Quinn, L.D., \& Holt, J.S. (2008). Ecological correlates of invasion by Arundo donax in three southern California riparian habitats. Biological Invasions, 10: 591-601.

60. Raunkiær, C. (1934). The Life Forms of Plants and Statistical Plant Geography, Oxford University Press, London. 632p

61. Reiffsteck, P. (2003). Recommandations pour l'utilisation des géosynthétiques dans la lutte contre l'érosion, Comité Français des Géosynthétiques. 128p

62. Reubens, B., Poesen, J., Danjon, F., Geudens, G., \& Muys, B. (2007). The role of fine and coarse roots in shallow slope stability and soil erosion control with a focus on root system architecture: a review. Trees, 21: 385-402 
63. Rey, F., Ballais, J., Marre, A., \& Rovéra, G. (2004). Rôle de la végétation dans la protection contre l'érosion hydrique de surface. Comptes Rendus Géoscience. 366: 991-998

64. Roose, E ., Sabir, M., Laouina, A., Benchakroun F., Karkouri., Lauri P., \& Qarro M (2010). Gestion durable des eaux et des sols au Maroc Valorisation des techniques traditionnelles méditerranéennes. IRD, 362p

65. Roose, E. (2002). Analyse du système des banquettes mécaniques : Propositions d'améliorations, de valorisation et d'évolution pour les gouvernorats de Kairouan, Siliana et Zaghouan, Tunisie, Tunis (TUN) ; Montpellier : Ministère de l'Agriculture ; IRD, $32 \mathrm{p}$

66. Roose E. (1994). Introduction à la gestion conservatoire de l'eau, de la biomasse et de la fertilité des sols (GCES). Bulletin Pédologique FAO 70 (Rome) : 420 p.

67. Saint Cast, C. (2019). Modélisation du développement architectural, de l'acclimatation au vent dominant et de l'ancrage du système racinaire du pin maritime. Thèse. Ecole Doctorale Science de l'Environnement. Université de Bordeaux. 214p

68. SAS (2004). SAS/STAT 9.1, User's guide, Institute Inc., Cary, NC, USA

69. Scippa, G.S., Di Michele, M., Di Iorio, A., Costa, A., Lasserre, B., \& Chiatante, D. (2005). The response of Spartium junceum roots to slope: Anchorage and gene factors. Annals of Botany, 97: 857-866

70. Selami, N., Auriac, M-C., Catrice, O., Capela, D., Kaid-Harche, M., \& Timmers T. (2014). Morphology and anatomy of root nodules of Retama monosperma (L.) Boiss. Plant and Soil, 379: 109-119

71. Silva, D. \& Beeson, R.C. Jr. (2011). A large-volume rhizotron for evaluating root growth under natural-like soil moisture conditions. HortScience 46:1677-1682

72. Stokes, A., Douglas, G.B., Fourcaud, T., Giadrossich, F., Gillies, C., Hubble, T., Kim, J.H., Loades, K.W., Mao, Z., McIvor, I.R., Mickovski, S.B., Mitchell, S., Osman, N., Phillips, C., Poesen, J., Polster, D., Preti, F., Raymond, P., Rey, F., Schwarz, M., Walker, L.R. (2014). Ecological mitigation of hillslope instability: ten key issues facing researchers and practitioners. Plant and Soil, 377:1-23

73. Stokes, A., Norris, J., Beek, L., Bogaard, T., Cammeraat, E., Mickovski, S., Jenner, A., Di Iorio, A., \& Fourcaud, T. (2008). How vegetation reinforces soil on slopes. In: Norris, JE, Stokes, A, Cammeraat, E, Van Beek, R, Nicoll, BC \& Achim, A. (Eds). Slope stability and erosion control, Ecotechnological solutions. Springer: Dordrecht, Pays-Bas. Pp: 65-118 
74. Tassin, C. (2012). Paysages végétaux du domaine méditerranéen: Bassin méditerranéen, Californie, Chili central, Afrique du Sud, Australie méridionale .Marseille : IRD Éditions, 428p.

75. Tribak, A., El Garouani, A., \& Abahrour, M. (2012). L'érosion hydrique dans les séries marneuses tertiaires du prérif oriental: agents, processus et évaluation quantitative. Revue Marocaine des Sciences Agronomiques et Vétérinaires.1:47-52

76. Vescovi, P. (1964). Etude comparée de l'ontogenèse de la grappe terminale indéfinie sans fleur terminale chez le Spartium junceum L. et du fonctionnement de l'apex du Lathyrus aphaca L. dans sa phase reproductrice, Bulletin de la Société Botanique de France, 111:173179

77. Vilela, A.E \& Ravetta, D. A. (2001). The effect of seed scarification and soil-media on germination, growth, storage, and survival of seedlings of five species of Prosopis L. (Mimosaceae). Journal of Arid Environments, 48: 171-184

78. Watson, A., Phillips, C., \& Marden, M. (1999). Root strength, growth, and rates of decay: root reinforcement changes of two tree species and their contribution to slope stability. Plant and Soil, 217: 39-47

79. Wu, T.H., McKinnell, III W.P., Swanston, D.N. (1979). Strength of tree roots and landslides on Prince of Wales Island, Alaska. Canadian Geotechnical Journal, 16: 19-33

80. Zappala, M.N. (2012). Biological effects of copper on Prosopis pubescens (screw bean mesquite). Thèse. Environmental Science and Engineering. University of Texas, El Paso. 153p

81. Zeh, H. (2007), Génie biologique, manuel de construction, Société suisse du génie biologique et Fédération Européenne pour le génie biologique.

82. Zunzunegui, M., Esquivias, M. P., Fernández-González, P., ValeraBurgos, J., Díaz Barradas, M. C., \& Gallego-Fernández, J. B. (2017). Morpho-physiological response of Retama monosperma to extreme salinity levels. Ecohydrology, 10: e1871 